\title{
A Study on the Thermodynamics of Grain Growth in R.F. Magnetron Sputtered NiO Thin Films
}

\author{
I. Dhanya ${ }^{1}$ and B. Sasi ${ }^{2}$ \\ ${ }^{1}$ Department of Physics, Catholicate College, Pathanamthitta, Kerala 689 645, India \\ ${ }^{2}$ Department of Physics, D.B. College, Sasthamcotta, Kollam, Kerala 690 521, India \\ Correspondence should be addressed to I. Dhanya; dhanya.iv@gmail.com
}

Received 22 March 2013; Revised 12 July 2013; Accepted 25 July 2013

Academic Editor: Mariana Braic

Copyright (c) 2013 I. Dhanya and B. Sasi. This is an open access article distributed under the Creative Commons Attribution License, which permits unrestricted use, distribution, and reproduction in any medium, provided the original work is properly cited.

\begin{abstract}
Postdeposition annealing of thin nickel films synthesized using R.F. magnetron sputtering technique is carried in this study. The XRD analysis indicates that annealing of the nickel films leads to the formation of nickel oxide with a preferential growth along (200) plane. The oxidation mechanism is observed with a phase transformation and results in polycrystalline NiO films. The surface morphology of the thin films was investigated by scanning electron microscopy (SEM) and atomic force microscopy (AFM) as a function of annealing temperature. The studies indicate the formation of well-defined grain boundaries due to agglomeration of nanocrystallites. The films annealed in the range $573-773 \mathrm{~K}$ are found to be porous. The optical transmission spectra of the films annealed at $773 \mathrm{~K}$ exhibit interference effects for photon energies below the fundamental absorption edge. The optical studies indicate the existence of direct interband transition across a bandgap of $3.7 \mathrm{eV}$ in confirmation with earlier band structure calculations.
\end{abstract}

\section{Introduction}

Nickel oxide $(\mathrm{NiO})$ is considered as a model p-type semiconductor. It is a wide bandgap $\left(E_{g} \sim 4 \mathrm{eV}\right)$ transition metal oxide, with a cubic rock-salt structure and antiferromagnetic properties below its Neel temperature $523 \mathrm{~K}$. Transparent conducting nickel oxide films have potential advantages for use as active layers in flat panel displays and gas sensor devices. There has been an increasing interest in developing nanostructured films due to their broad range of applications as catalysts, electrochromic display devices, and fuel cells [15]. P-type conducting thin films are required for the fabrication of many optoelectronic devices, which make use of hole injection [6]. Nickel oxide, as one of the relatively few p-type metal oxides, has got considerable attention because of its stable wide bandgap and excellent chemical stability [7]. Recent studies have shown that $\mathrm{NiO}$ thin films can make attractive sensing materials in gas like hydrogen and humidity detection devices [8]. There are several reports on nickel oxide thin film preparation by physical vapor deposition [9], electron beam evaporation [10], dip coating [11], sputtering [12-14], spray pyrolysis, chemical vapor deposition, sol gel, and pulsed laser deposition [15]. R.F. magnetron sputtering has been used extensively to fabricate high surface area thin films, having large potential in the region of gas sensing [16]. Attempt has been made in this study to prepare $\mathrm{NiO}$ films by postdeposition annealing of R.F. magnetron sputtered thin nickel films and to understand the influence of postdeposition heat treatment on the crystalline phase formation, surface morphology, and optical properties of the film using different structural, optical, and electrical characterization tools.

\section{Experimental Details}

Thin nickel films are prepared by R.F. magnetron sputtering in a plasma focusing magnetic field using $99.99 \%$ pure nickel powder as the target, purchased from Sigma-Aldrich company. Clean soda lime glass slides are used as substrate material and kept perpendicular to the target surface at a distance of $4.5 \mathrm{~cm}$. The sputter deposition is performed using R.F. magnetron sputtering equipment (Hind Hivac. Planar magnetron sputtering, model-12" MSPT) with a frequency of $13.56 \mathrm{MHz}$ and an R.F. sputtering power of $200 \mathrm{~W}$. 
TABLE 1: Sample codes with preparation conditions.

\begin{tabular}{lccccccccc}
\hline Sample code & S1 & S2 & S3 & S4 & S5 & S6 & S7 & S8 & S9 \\
\hline Dep. time (Minutes) & 45 & 45 & 45 & 45 & 45 & 15 & 15 & 15 \\
Annealing temp. (K) & 413 & 473 & 573 & 773 & 773 & 413 & 473 & 573 & 773 \\
Annealing time (h) & 0 & 3 & 3 & 3 & 5 & 0 & 3 & 3 \\
\hline
\end{tabular}

Before sputtering, the chamber is evacuated to a base pressure of $10^{-6} \mathrm{mbar}$. The reflected power is minimized to $0 \mathrm{~W}$ and the argon inlet is adjusted to get a work pressure of $4 \times 10^{-2}$ mbar in the chamber during deposition. The films are then deposited for time durations of 45 and 15 minutes [17]. In order to study the oxidation process, the films prepared are thermally annealed in air at constant temperatures within the range of 473 to $773 \mathrm{~K}$ for 3 hours in a programmable muffle furnace. One sample is annealed to $773 \mathrm{~K}$ for a longer period of 5 hours. The thin film samples prepared under different conditions together with sample codes assigned for convenience are listed in Table 1.

The crystalline structural changes of the sputtered films were determined by X-ray diffraction (XRD) in reflection geometry with a Philips P W 1710 diffractometer using $\mathrm{Cu} K_{\alpha}$ radiation $(0.15406 \mathrm{~nm})$. The XRD patterns were recorded at a scanning rate of $0.05 \mathrm{deg} \mathrm{s}^{-1}$ in the angular $(2 \theta)$ ranges from $15^{\circ}$ to $65^{\circ}$. Film structure and surface morphology were investigated using atomic force microscopy (AFM) in contact mode with digital instruments Nanoscope III and scanning electron microscopy (SEM) using the system Quanta 200, fitted with an energy dispersive X-ray spectroscope (EDX). The stoichiometric composition of the film was evaluated by energy dispersive $\mathrm{X}$-ray analysis. Optical measurements were performed in the wavelength ranging from 300 to $900 \mathrm{~nm}$ using a double beam UV-Visible spectrophotometer, JASCO-V550. The thickness of films was determined from the oscillations in the transmission spectra. The resistivity of the samples is measured by the two probe method employed with a Keithley digital source meter model 6430.

\section{Results and Discussion}

3.1. XRD Studies. In order to investigate the dependence of crystalline structural changes on annealing, the X-ray diffractograms of the samples annealed at different conditions are taken and are shown in Figure 1.

Only the crystalline phases of the fcc structure of $\mathrm{Ni}$ and the rock salt of $\mathrm{NiO}$ were identified. The peaks are consistent with the respective ASTM data and indicate the formation of $\mathrm{NiO}$ with a preferential growth along (200) plane. The peaks obtained at $2 \theta$ equals to $44.55^{\circ}$ and $51.91^{\circ}$ represent diffractions corresponding to (111) and (200) planes of nickel. These results do not show any formation of the $\mathrm{Ni}_{2} \mathrm{O}_{3}$ phase as that has been reported to appear in the temperature range of $573-773 \mathrm{~K}$ for evaporated nickel films [9]. It can be seen that the intensity of (200) peak corresponding to $\mathrm{NiO}$ increases with increasing annealing temperature. This is due to enhanced oxidation kinetics and improvements

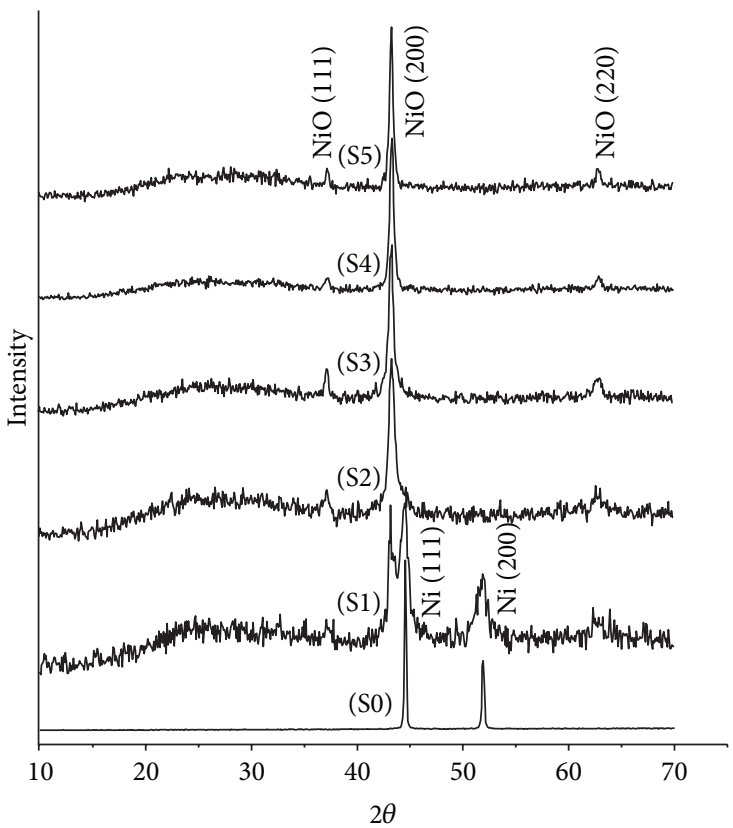

FIGURE 1: XRD pattern of Ni powder (S0), Ni/NiO thin film samples (S1, S2, S3, S4, and S5) peak positions for S0Ni powder, and Ni thin film at $\mathrm{Ni}(111)$ and $\mathrm{Ni}(200)$. For $\mathrm{NiO}$ thin films S2, S3, S4, and S5 at $\mathrm{NiO}$ (111), $\mathrm{NiO}(200)$, and $\mathrm{NiO}(220)$.

in crystallinity. The better crystallinity of films at higher temperature is in agreement with previous studies [18-20]. The full width at half maximum (FWHM) of the peak is found to decrease, and the peaks become sharp with increase of annealing temperature. This indicates that the $\mathrm{NiO}$ average grain size increases with heat treatment. For lower annealing temperatures, the samples obtained are of imperfect lattice structure and have smaller grain size.

The crystallite size can be calculated using Scherrer's equation as follows:

$$
D=\frac{K \lambda}{B \cos \theta},
$$

where $D$ is the diameter of the nanoparticles, $K=0.9, \lambda$ is the wavelength of X-rays, $\theta$ is the Bragg's angle of XRD peak, and $B$ is the FWHM for the peak [15].

The crystallite size determined from the (200) diffraction peak for the samples as a function of annealing temperature and time is given in Table 2. It must be mentioned that for the deposited film, it was not possible to obtain a reliable value of $\mathrm{NiO}$ grain size. 
TABLE 2: Lattice parameters of annealed $\mathrm{NiO}$ thin films with respect to Ni powder.

\begin{tabular}{lcccrc}
\hline Parameter & Ni powder & S2 & S3 & S4 & S5 \\
\hline$a_{111}$ & 4.174 & 4.183 & 4.1829 & 4.161 & 4.160 \\
$a_{200}$ & 4.176 & 4.166 & 4.162 & 4.168 & 4.168 \\
$a_{220}$ & - & 4.186 & 0.178 & 0.016 & 0.011 \\
$\Delta a_{(220-200)}$ & - & 0.002 & 0.12 & 0.0073 \\
$\Delta a_{(200-111)}$ & 0.002 & 0.017 & 15 & 16 & 0.010 \\
Grain size $(\mathrm{nm})$ & 80 & 14 & & 0.006 \\
\hline
\end{tabular}
ing:

The lattice parameter can be calculated using the follow-

$$
a_{(h k l)}=d_{(h k l)}\left(\sqrt{h^{2}+k^{2}+l^{2}}\right),
$$

where $h, k$, and $l$ are all integers, $(h k l)$ is the lattice plane index, and $a_{(h k l)}$ is the corresponding lattice constant [21]. The lattice constants of oxidized nickel samples obtained are listed in Table 2. Even though $\mathrm{NiO}$ is of rock salt structure, the presence of large number of vacant lattice sites and local lattice disorders may lead to obvious lattice distortions and reduction in intensities or even the disappearance of XRD peaks of some lattice planes [22].

The lattice parameter " $a$ " and change in lattice parameter " $\Delta a$ " along the planes of S2, S3, and S4 are found using the relation following:

$$
\Delta a=a_{111}-a_{200} \cong a_{200}-a_{220} .
$$

It is found that there are changes in lattice parameters of the annealed $\mathrm{NiO}$ thin film samples when compared with that of $\mathrm{NiO}$ powder. The slit difference in peaks of $\mathrm{Ni}$ powder with respect to annealed $\mathrm{NiO}$ thin films is due to the film preparation conditions and annealing effects [23]. This implies that the prepared nanocrystalline $\mathrm{NiO}$ thin film samples contain a large number of vacant lattice sites, vacancy clusters, and local lattice disorders. The change in unit cell parameters $\Delta a$ along (111), (200), and (220) plane, for different low-temperature samples is almost the same and collectively leads to the cubic crystalline nature of $\mathrm{NiO}$. The variation of lattice parameters with temperature is shown in Figure 2.

The study of texture grade (Tc) for different planes is helpful in determining the crystalline nature of samples under different preparation conditions. The theoretical intensities for texture grade are taken from JCPDS file 4-835 of cubic $\mathrm{NiO}$. The texture grade of samples is given by

$$
\mathrm{Tc}_{I(h k l)}=\frac{I_{(h k l)} / I_{(h k l)}^{0}}{1 / k \sum_{i=1}^{k} I_{(h k l)} / I_{(h k l)}^{0}} .
$$

XRD patterns of different samples in Figure 1 shows prominent peaks along (200), (111), and (220) planes; thus, the value of reflection number $K$ is taken as 3. Among the peaks, prominent one is that of 200 plane having $I_{(h k l)}^{0}=100 \%$, with (111) of $91 \%$ and (220) of 57\% from JCPDS file, and $I_{(h k l)}$ represents the intensities of the corresponding peaks,

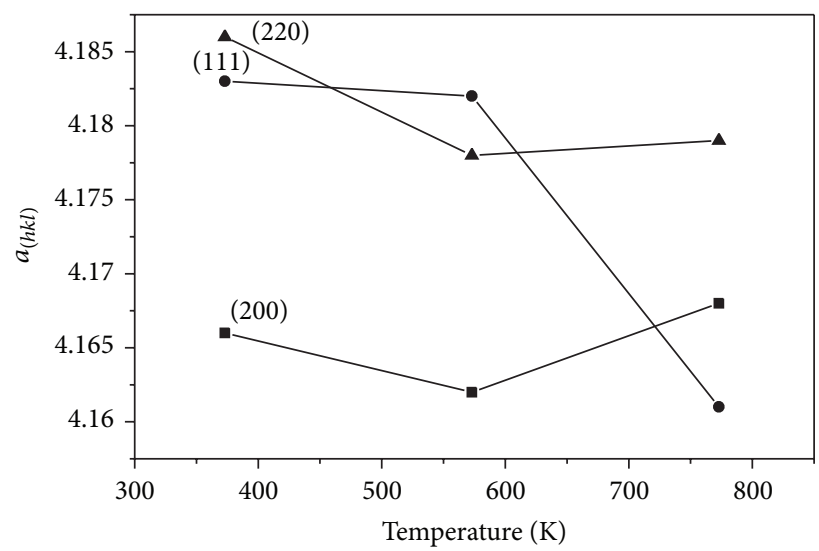

Figure 2: Variation of lattice parameters with temperature.

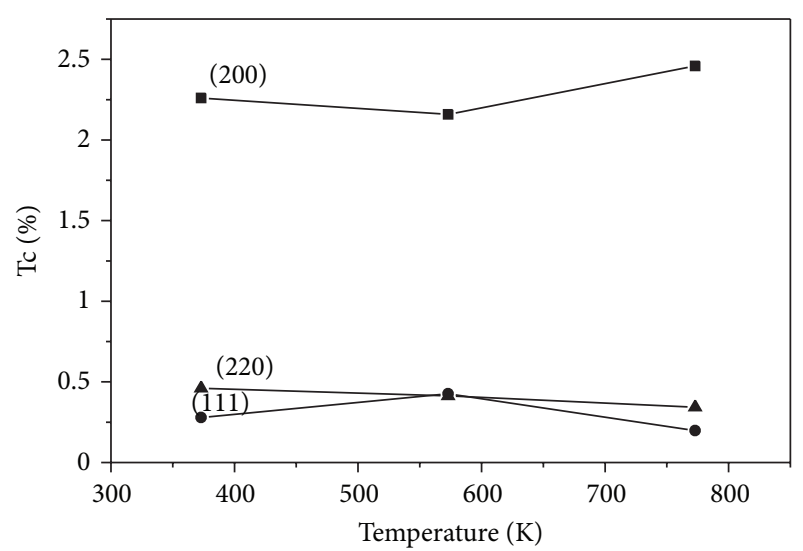

FIGURE 3: Annealing effect on texture grade of $a_{200}, a_{220}$, and $a_{111}$ plane.

in the diffraction patterns for different samples. The texture grade for $\mathrm{NiO}$ peak along (200) plane shows a polycrystalline nature. For annealed samples, the texture grade shows a deviation from unity as shown in Figure 3.

The annealed samples with preferential orientation along (200) plane show values greater than one which implies the texture nature as well. The same annealed samples with peak orientations along (111) and (220) plane indicate "Tc" values smaller than unity, which means there is a depletion of grains in this direction [24]. 


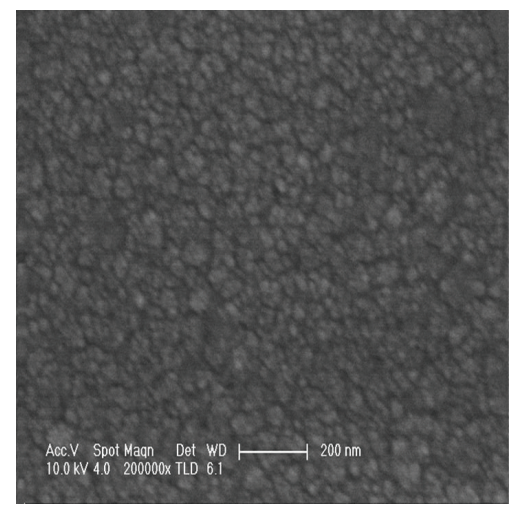

(a)

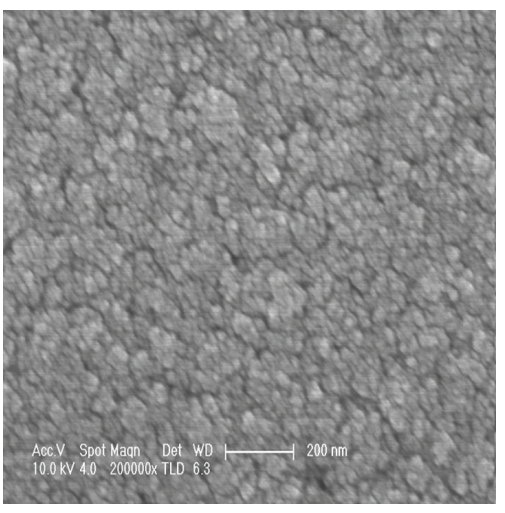

(b)

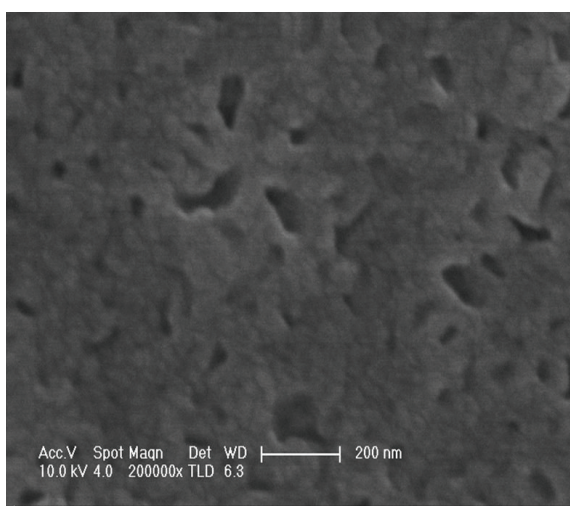

(c)

FIgURE 4: SEM micrographs of NiO thin film samples (a) S1, (b) S3, (c) S5.

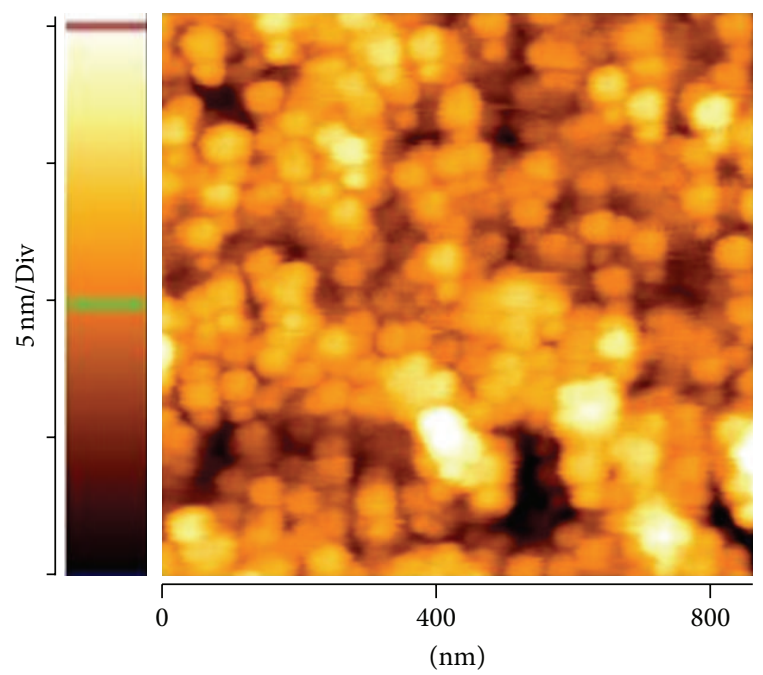

Figure 5: AFM image of $\mathrm{NiO}$ thin film sample (S4).

3.2. SEM and AFM Studies. The SEM micrographs of nickel films deposited for 45 minutes (S1) and subjected to annealing temperatures of $573 \mathrm{~K}$ for $3 \mathrm{~h}$ (S3), and $773 \mathrm{~K}$ for $5 \mathrm{~h}$ (S5) are shown in Figure 4. The surface morphology of a deposited film in Figure 4(a) consists of closely packed nanocrystals of $\mathrm{Ni} / \mathrm{NiO}$. Further increase of annealing temperature and time gives rise to porous film with voids between the grains (Figure 4(c)). The AFM image of nickel oxide film (sample S3) is shown in Figure 5.

The AFM image of sample annealed at $773 \mathrm{~K}$ for 3 hours (S4) indicates the agglomeration of nanocrystals and the voids in between the grains. The peaks in between vacant sites in the AFM image account for the high concentration of nucleation centers while annealing the film. The characteristics of height profile indicate the smooth and uniform nature of the film surface on annealing.

3.3. X-Ray Energy Dispersive Analysis. Energy dispersive spectroscopy was done for the elemental analysis of the film

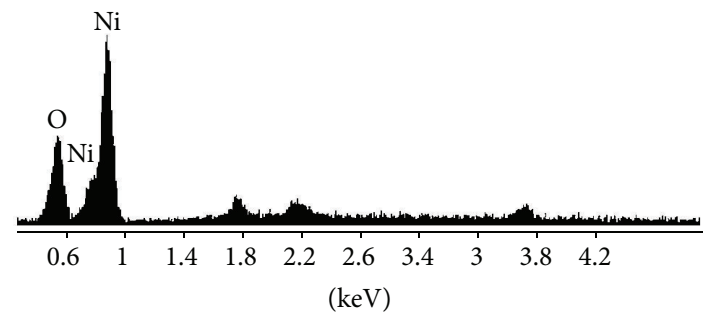

FIGURE 6: Energy dispersive spectroscopy of Ni film coated for 45 minutes and annealed at $773 \mathrm{~K}$ for 5 hours.

surface. The EDX spectrum of the film annealed at $773 \mathrm{~K}$ for $5 \mathrm{~h}$ is shown in Figure 6.

There are no other prominent peaks besides those corresponding to nickel and oxygen detected in the spectrum. The nonstoichiometric atomic ratio $(\mathrm{Ni} / \mathrm{O}-70 / 30)$ shows the effect of controlled oxygen mechanism on annealing.

3.3.1. Optical and Electrical Studies. The transmission spectra of samples S4, S5, S8, and S9 in the wavelength range 300$900 \mathrm{~nm}$ are shown in Figure 7. The color of the film in the deposited state is opaque, dark brown, and has negligible transmittance. On other hand, samples on annealing in air showed a color change and an increase in transmittance depending on annealing temperature. Similar reports of color change in $\mathrm{NiO}$ films with preparation conditions are found in literature $[5,9,25]$. The optical transmission spectra of the films annealed at $773 \mathrm{~K}$ exhibit interference effects for photon energies below the fundamental absorption edge. This arises due to difference of refractive index of the film with the substrate and interference of multiple reflections originated from films and substrate surfaces.

The optical bandgap $E_{g}$ of the films can be determined from the transmission spectra by the Tauc plot [26] using the following relation

$$
\alpha h v=A\left(h v-E_{g}\right)^{1 / 2}
$$

where $A$ is a constant and $h \nu$ is the incident photon energy. The variation of $(\alpha h \nu)^{2}$ versus $h \nu$ for the $\mathrm{NiO}$ samples is 


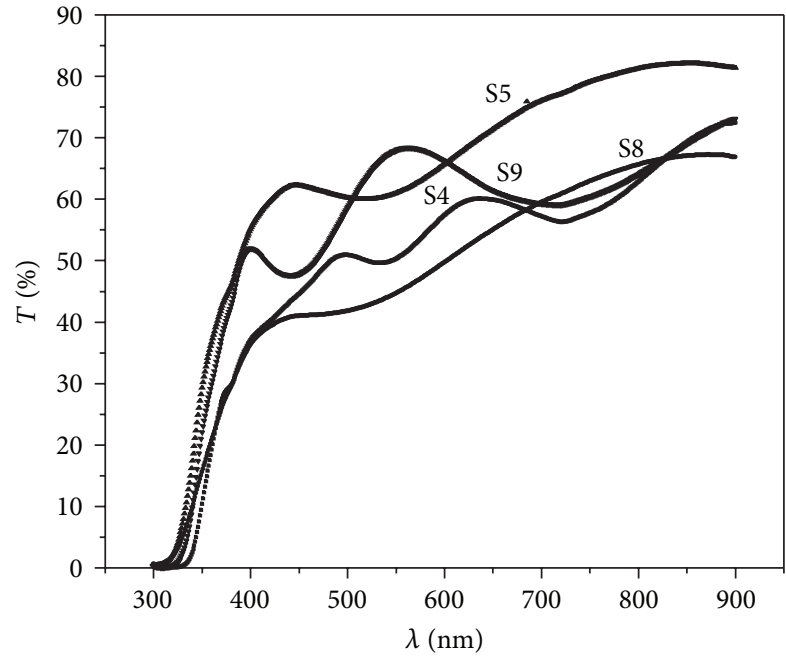

Figure 7: Transmission spectra of different thin film samples.

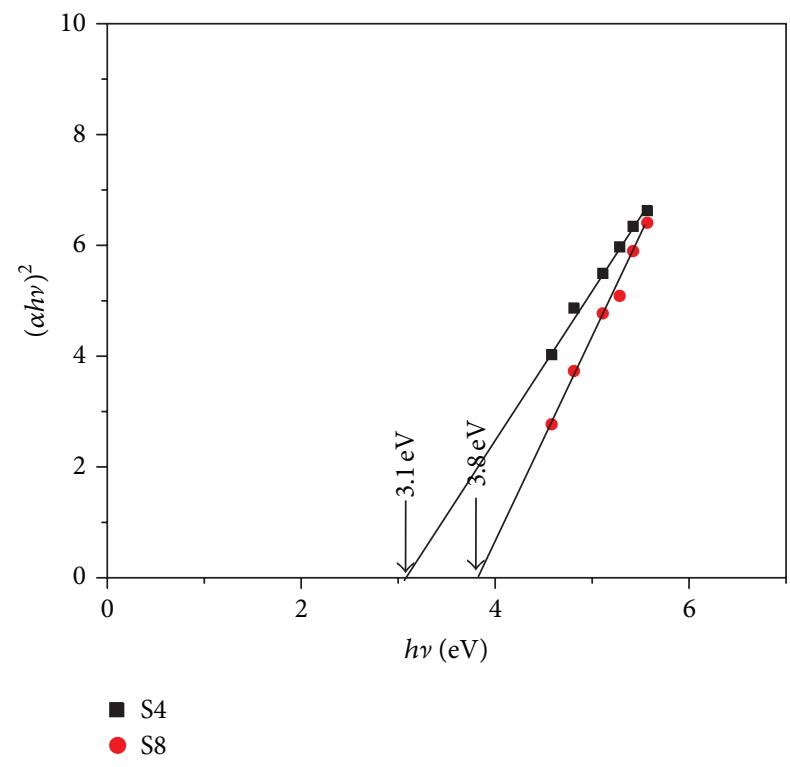

FIgURE 8: Plot of $(\alpha h \nu)^{2}$ versus $h v$ for $\mathrm{NiO}$ thin film samples $\mathrm{S} 4$ and S8.

shown in Figure 8. The nature of the plots indicates the existence of direct interband transition in these $\mathrm{NiO}$ films [27]. The bandgap of the films is determined by extrapolating the linear portion of the plot to the energy axis. It is observed that the band gap energy gets shifted towards lower energy values and the slope of the plot decreases with increase in deposition time and annealing. The values of $E_{g}$ for nickel film annealed at $773 \mathrm{~K}$ for $3 \mathrm{~h}$ with a sputter deposition of 45 and 15 minutes are estimated to be 3.1 and $3.8 \mathrm{eV}$, respectively. The values indicate that the optical bandgap of the films are sensitive to deposition time. Reported bandgap energies for $\mathrm{NiO}$ films are in the range of 3.6 to $4.0 \mathrm{eV}$ [28]. The electrical conductivity of $\mathrm{NiO}$ films depends on their structure, composition, and deposition environment. The sample S6 is opaque, is dark brown, and shows a sheet resistance of $825 \mathrm{~K} \Omega / \square$.
The conductivity is found to increase with sputter deposition time (for S1, sheet resistance is $387 \mathrm{~K} \Omega / \square$ ) and indicate the presence of a large number of $\mathrm{Ni}^{3+}$ ions in film which in turn enhanced the p-type conductivity. The film annealed at $773 \mathrm{~K}$ for $5 \mathrm{~h}$ (S5) is found to have a sheet resistance of $125 \mathrm{M} \Omega / \square$ and shows a maximum transmittance of $80 \%$ in the visible region.

\section{Conclusions}

Thin nickel films are prepared at room temperature by R.F. magnetron sputtering technique from pure nickel powder source. The effect of annealing temperature and deposition time on the properties of these films has been investigated. The NiO films obtained showed enhanced transparency and high surface uniformity. The structural analysis using Xrays reveals the formation of cubic $\mathrm{NiO}$ with a well-defined preferential growth along (200) plane. The decrease of full width at half maximum (FWHM) and sharpness of peaks with increase of annealing temperature show that average grain size of $\mathrm{NiO}$ thin films increase due to heat treatment. The average grain size decreases with annealing and it shows a microlevel nature on thin films with respect to nickel powder sample. Texture grade analysis on $\mathrm{NiO}$ thin films shows its deviation from unity that leads to polycrystalline nature in its prominent peak (200) and a depletion of grains along (220) and (111) peaks. Annealing effects on the SEM and AFM studies on thin film result in the increase of oxidation kinetics, grain rotations, and enhanced crystallization which in turn produces nanocrystalline $\mathrm{NiO}$ film with well-defined grain boundaries. AFM analysis on annealed thin nickel film results in an agglomeration of $\mathrm{NiO}$ nanocrystals with voids in between grains. The controlled oxidation mechanism is well explained by the nonstoichiometric composition rates of $\mathrm{Ni}$ and oxygen in EDX analysis. The fringes on the transmission spectra are a measure of the thickness uniformity and high optical quality of the film. The annealed sample S5 shows a high transparency of $80 \%$ with appreciable sheet resistance. The bandgap energies for $\mathrm{NiO}$ samples $\mathrm{S} 4$ and $\mathrm{S} 8$ have comparable values with the reported ones. The conductivity was found to increase more with sputter deposition time than annealing.

\section{References}

[1] S. Passerini and B. Scrosati, "Electrochromism of thin-film nickel oxide electrodes," Solid State Ionics, vol. 53-56, no. 1, pp. 520-524, 1992.

[2] G. Wakefield, P. J. Dobson, Y. Y. Foo, A. Loni, A. Simons, and J. L. Hutchison, "The fabrication and characterization of nickel oxide films and their application as contacts to polymer/porous silicon electroluminescent devices," Semiconductor Science and Technology, vol. 12, no. 10, pp. 1304-1309, 1997.

[3] D. Adler and J. Feinleibi, "Electrical and optical properties of narrow-band materials," Physical Review B, vol. 2, pp. 3112-3134, 1970.

[4] E. L. Miller and R. E. Rochilean, "Electrochemical and electrochromic behavior of reactively sputtered nickel oxide," Journal of the Electrochemical Society, vol. 144, no. 6, pp. 1995-2003, 1997. 
[5] H. Sato, T. Minami, S. Takata, and T. Yamada, “Transparent conducting p-type $\mathrm{NiO}$ thin films prepared by magnetron sputtering," Thin Solid Films, vol. 236, no. 1-2, pp. 27-31, 1993.

[6] Y. Tawada, H. Okamoto, and Y. Hamakawa, " $a-\mathrm{SiC}: \mathrm{H} / a-\mathrm{Si}: \mathrm{H}$ heterojunction solar cell having more than $7.1 \%$ conversion efficiency," Applied Physics Letters, vol. 39, no. 3, p. 237, 1981.

[7] B. Sasi and K. G. Gopchandran, "Nanostructured mesoporous nickel oxide thin films," Nanotechnology, vol. 18, no. 11, Article ID 115613, 2007.

[8] I. Fasaki, A. Giannoudakos, M. Stamataki et al., "Nickel oxide thin films synthesized by reactive pulsed laser deposition: characterization and application to hydrogen sensing," Applied Physics A, vol. 91, no. 3, pp. 487-492, 2008.

[9] B. Sasi, K. G. Gopchandran, P. K. Manoj, P. Koshy, P. Prabhakara Rao, and V. K. Vaidyan, "Preparation of transparent and semiconducting NiO films," Vacuum, vol. 68, no. 2, pp. 149-154, 2002.

[10] P. Lunkenheimer, A. Loidl, C. R. Ottermann, and K. Bange, "Correlated barrier hopping in NiO films," Physical Review B, vol. 44, no. 11, pp. 5927-5930, 1991.

[11] A. J. Varkey and A. F. Fort, "Solution growth technique for deposition of nickel oxide thin films," Thin Solid Films, vol. 235, no. 1-2, pp. 47-50, 1993.

[12] J. S. E. M. Svensson and C. G. Granqvist, "Electrochromic hydrated nickel oxide coatings for energy efficient windows: optical properties and coloration mechanism," Applied Physics Letters, vol. 49, no. 23, p. 1566.

[13] S. Yamada, T. Yoshioka, M. Miyashita, K. Urabe, and M. Kitao, "Electrochromic properties of sputtered nickel-oxide films," Journal of Applied Physics, vol. 63, no. 6, pp. 2116-2119, 1988.

[14] A. Iida and R. Nishikawa, "A thin film of an Ni-NiO heterogeneous system for an optical recording medium," Japanese Journal of Applied Physics, vol. 33, pp. 3952-3959, 1994.

[15] B. Sasi and K. G. Gopchandran, "Preparation and characterization of nanostructured $\mathrm{NiO}$ thin films by reactive-pulsed laser ablation technique," Solar Energy Materials and Solar Cells, vol. 91, no. 15-16, pp. 1505-1509, 2007.

[16] Y. M. Lu, W. S. Hwang, J. S. Yang, and H. C. Chuang, "Properties of nickel oxide thin films deposited by RF reactive magnetron sputtering," Thin Solid Films, vol. 420-421, pp. 54-61, 2002.

[17] H. Sato, T. Minami, S. Takata, and T. Yamada, "Transparent conducting p-type $\mathrm{NiO}$ thin films prepared by magnetron sputtering," Thin Solid Films, vol. 236, no. 1-2, pp. 27-31, 1993.

[18] I. Hotovy, J. Huran, L. Spiess, J. Liday, H. Sitter, and Š. Hašcík, "The influence of process parameters and annealing temperature on the physical properties of sputtered $\mathrm{NiO}$ thin films," Vacuum, vol. 69, no. 1-3, pp. 237-242, 2002.

[19] F. F. Ferreira, M. H. Tabacniks, M. C. A. Fantini, I. C. Faria, and A. Gorenstein, "Electrochromic nickel oxide thin films deposited under different sputtering conditions," Solid State Ionics, vol. 86-88, no. 2, pp. 971-976, 1996.

[20] J.-L. Yang, Y.-S. Lai, and J. S. Chen, "Effect of heat treatment on the properties of non-stoichiometric p-type nickel oxide films deposited by reactive sputtering," Thin Solid Films, vol. 488, no. 1-2, pp. 242-246, 2005.

[21] C. Kittel, "Indirect Exchange Interactions in Metals," Solid State Physics, vol. 22, pp. 1-26, 1969.

[22] Z. W. Chen, J. K. L. Lai, and C. H. Shek, "Insights into microstructural evolution from nanocrystalline $\mathrm{SnO}_{2}$ thin films prepared by pulsed laser deposition," Physical Review B, vol. 70, no. 16, Article ID 165314, 2004.
[23] I. Dhanya and C. S. Menon, "Annealing effects on electrical, optical and structural properties of semiconducting transparent tetra-tert-butyl 2,3 naphthalocyanine thinfilms," Journal of NonCrystalline Solids, vol. 357, no. 21, pp. 3631-3636, 2011.

[24] I. Hotovy, J. Huran, and L. Spiess, "Characterization of sputtered $\mathrm{NiO}$ films using XRD and AFM," Journal of Materials Science, vol. 39, no. 7, pp. 2609-2612, 2004.

[25] P. Puspharajah, S. Radhakrishna, and A. K. Arof, "Transparent conducting lithium-doped nickel oxide thin films by spray pyrolysis technique," Journal of Materials Science, vol. 32, no. 11, pp. 3001-3006, 1997.

[26] J. Tauc, Amorphous and Liquid Semiconductors, Plennm, London, UK, 1974.

[27] J. I. Panaove, Optical Processes in Semiconductors, Prentice Hall, Upper Saddle River, NJ, USA, 1971.

[28] A. B. Kunz, "Electronic structure of NiO," Journal of Physics C, vol. 14 , no. 16, p. 445, 1981. 

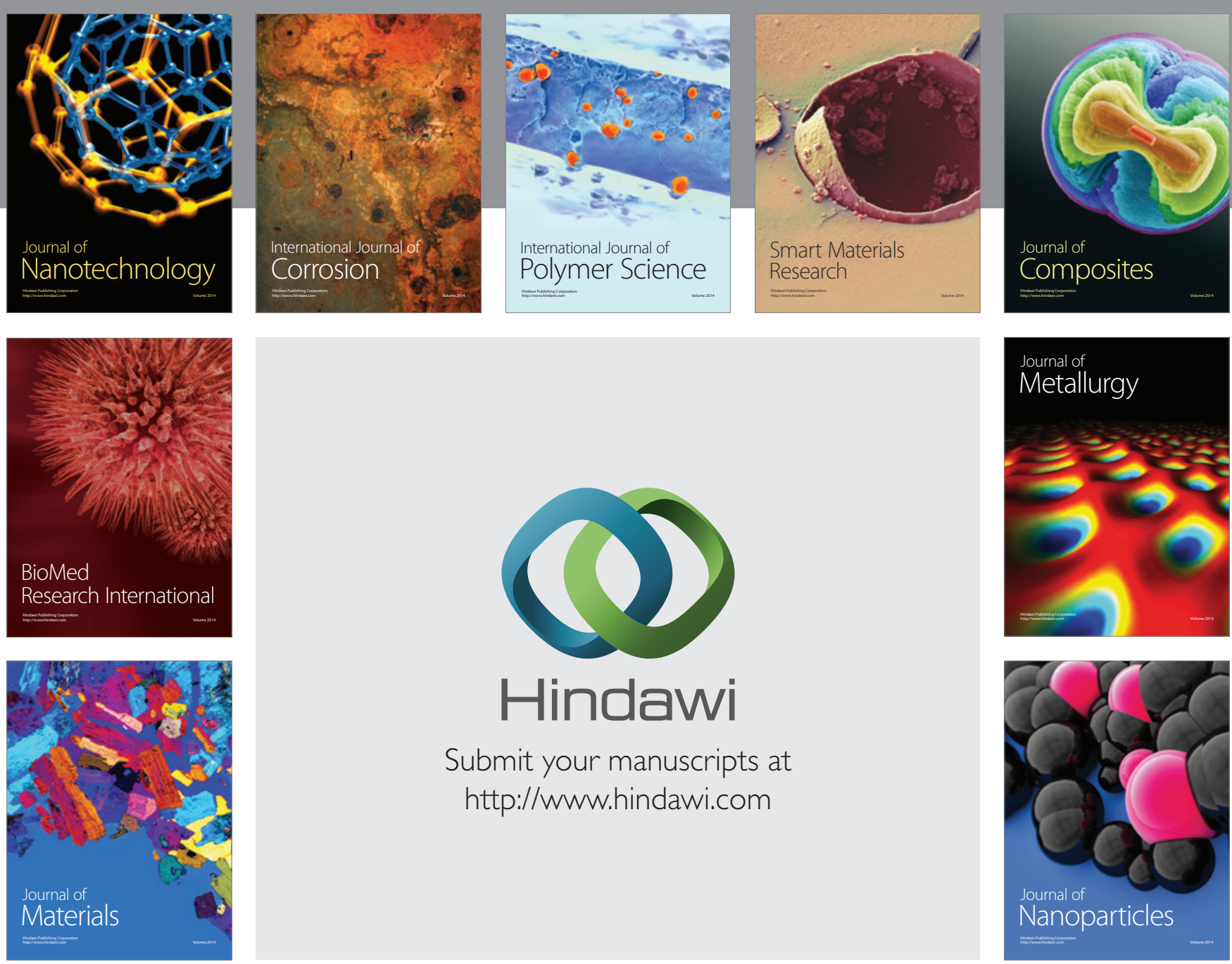

Submit your manuscripts at http://www.hindawi.com
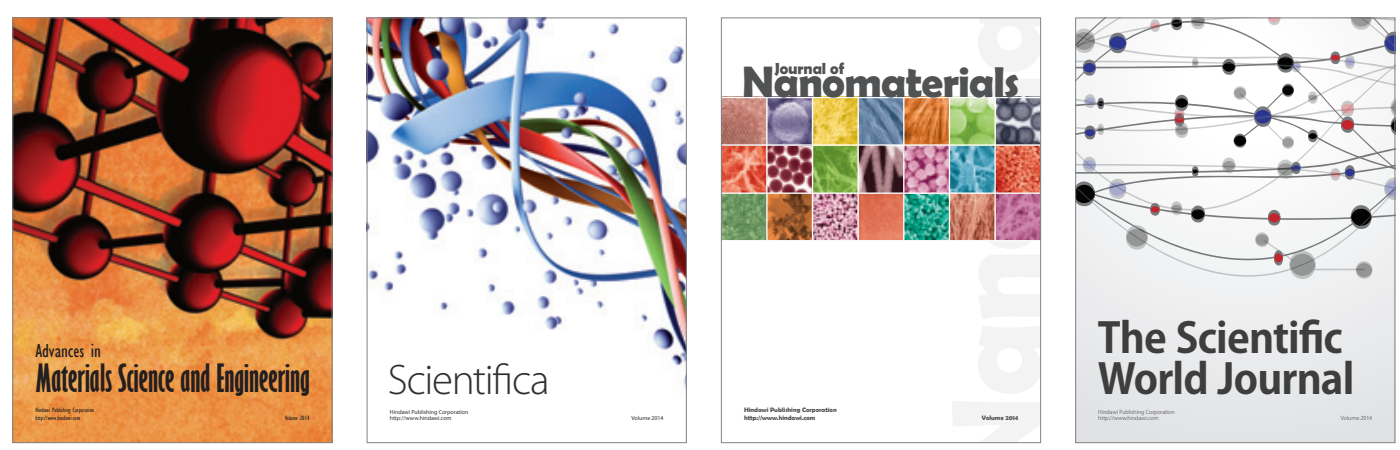

\section{The Scientific World Journal}
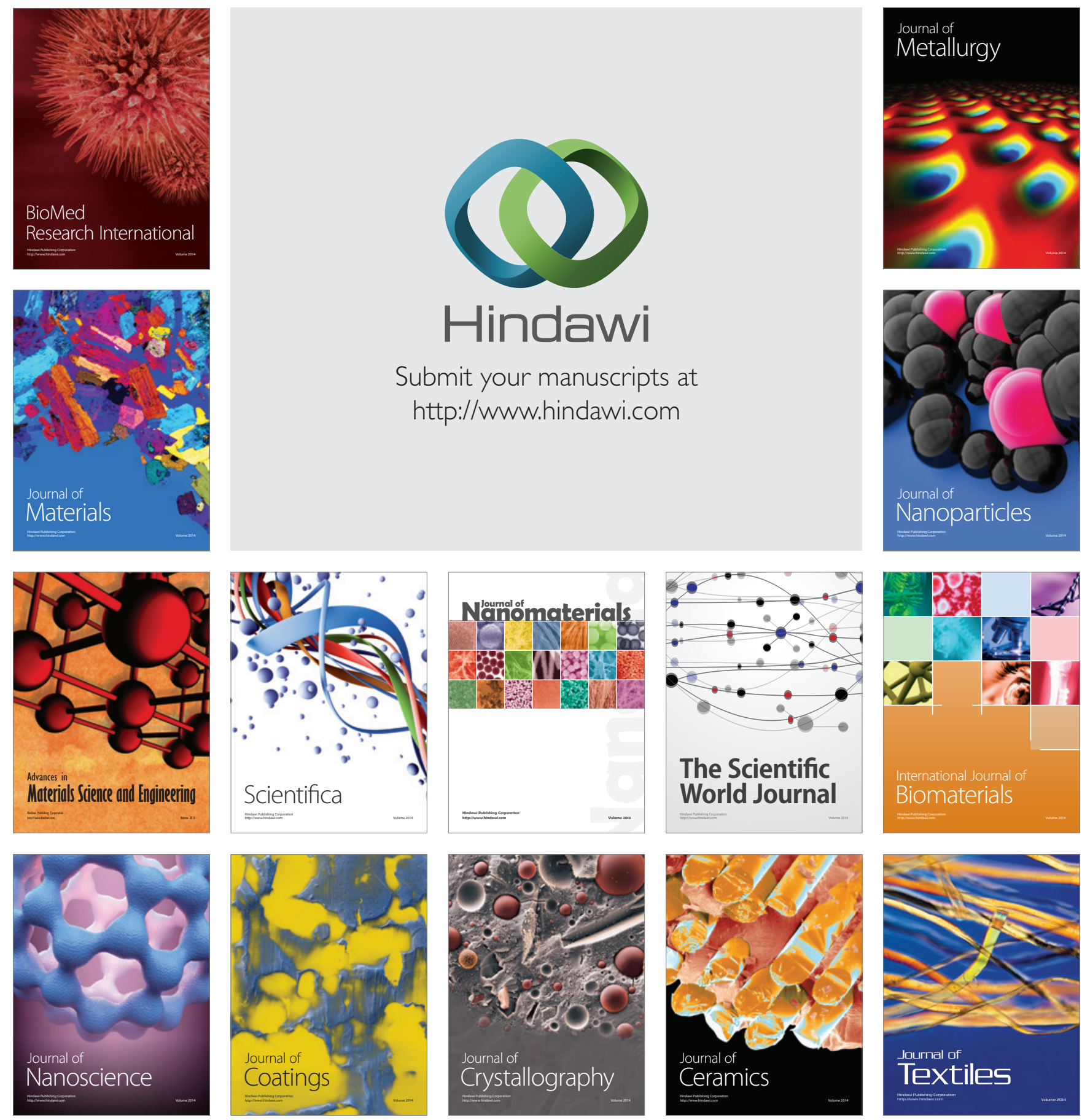